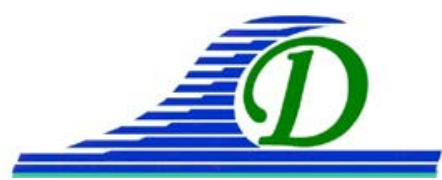

XIII ${ }^{\text {èmes }}$ Journées Nationales Génie Côtier - Génie Civil

Dunkerque, 2-4 juillet 2014

DOI:10.5150/jngcgc.2014.100 C Editions Paralia CFL

disponible en ligne - http://www.paralia.fr - available online

\title{
Méthodologie de caractérisation de la sensibilité des sols aux processus d'érosion interne
}

\author{
Didier MAROT ${ }^{1}$, Abdul ROCHIM ${ }^{1}$, Hong Hai NGUYEN ${ }^{2}$, \\ Fateh BENDAHMANE ${ }^{1}$, Luc SIBILLE ${ }^{3}$
}

\section{Université de Nantes, Institut GeM, UMR CNRS 6183,}

58 rue Michel Ange, BP 420, 44606 Saint-Nazaire, France.

didier.marot@univ-nantes.fr ; abdoul.rochim@etu.univ-nantes.fr ;

fateh.bendahmane@univ-nantes.fr

2. Université de Danang, Faculté d'Ingénierie des Ponts et Chaussées,

54 Nguyen Luong Bang Str, Danang, Vietnam.

honghaidhbk@yahoo.com

3. Université de Grenoble, Laboratoire3SR, UMR CNRS 5521,

Domaine Universitaire, BP53, 38041 Grenoble Cedex 9, France.

luc.sibille@3sr-grenoble.fr

\section{Résumé :}

L'érosion interne est responsable de nombreuses instabilités d'ouvrages hydrauliques en terre, tant en domaine fluvial qu'en domaine côtier. Deux types d'érosion interne peuvent être distingués: l'érosion de volume ou suffusion, qui ne mobilise que la fraction fine du sol et l'érosion d'interface qui peut mobiliser tous les grains. Dans la littérature, la caractérisation de la suffusion a été essentiellement réalisée par l'élaboration de critères d'évaluation de l'initiation du processus. Ces critères peuvent s’avérer conservatifs et la comparaison des critères granulométriques met en évidence la nécessité de choisir le critère en fonction de la distribution granulométrique du sol étudié. Par ailleurs la détermination d'un gradient hydraulique critique d'initiation de l'érosion est fortement influencée par la taille des échantillons testés. Afin de s'affranchir de ces effets d'échelle et de prendre en compte l'historique de chargement hydraulique, l'approche proposée consiste à déterminer l'énergie dissipée par le fluide lors de son écoulement interstitiel. Pour caractériser la sensibilité à l'érosion d'interface des sols, un indice de résistance à l'érosion a été proposé et a permis l'obtention d’une classification unique pour les essais de Hole Erosion Test et de Jet Erosion Test.

L'ensemble de cette étude nous permet alors de proposer une méthodologie systématique pour la caractérisation de la sensibilité des sols aux différents processus d'érosion interne.

Mots-clés : Erosion interne, Suffusion, Erosion d'interface, HET, JET, Effet d'échelle, Energie, Classification de sensibilité à l'érosion. 


\section{Introduction}

\subsection{Contexte général}

L'érosion interne est responsable de nombreuses instabilités d'ouvrages hydrauliques en terre. Sont notamment concernées des levées et des digues, tant en domaine fluvial qu'en domaine côtier. Selon la terminologie établie dans le cadre du projet ERINOH (2014), l'érosion interne peut être décrite suivant deux types : l'érosion de volume ou suffusion, qui ne mobilise que la fraction fine du sol et l'érosion d'interface qui peut mobiliser tous les grains.

\subsection{Caractère conservatif des critères granulométriques d'initiation de la suffusion}

Dans la littérature, la caractérisation de la suffusion a été essentiellement réalisée par l'élaboration de critères d'évaluation de l'initiation du processus qui reposent principalement sur l'analyse de la courbe granulométrique du sol. WAN et FELL (2008) ainsi que LI et FANNIN (2008) ont montré qu'en fonction de la courbe granulométrique des sols, les critères granulométriques les plus couramment utilisés peuvent s'avérer conservatifs. Il convient donc d'identifier les critères adaptés à chaque type de distribution granulométrique. Par ailleurs, le non respect d'un critère granulométrique n’implique pas nécessairement une forte sensibilité à la suffusion à cause des forces de contact entre les grains qui s’opposent à leur détachement. Nous pouvons donc penser que la vérification de critères granulométriques permet de déterminer uniquement la susceptibilité du sol à être suffusif. D'autres paramètres doivent être pris en compte pour caractériser la sensibilité à la suffusion, notamment la porosité du sol qui dépend elle-même du chargement mécanique que subit le sol, la forme des grains (MAROT et al., 2012a) et la sollicitation hydraulique.

\subsection{Effet d'échelle sur l'interprétation de l'initiation de l'érosion}

Afin de prendre en compte la sollicitation hydraulique ainsi que le chargement mécanique subis par le sol, LI (2008) a exprimé le gradient hydraulique critique d'initiation de la suffusion par :

$i_{c r}=\alpha\left(\frac{\sigma_{t 0}^{\prime}}{\gamma_{w} \Delta z}+\frac{\gamma^{\prime}}{\gamma_{w}}\right)$

avec $\alpha$ : facteur de réduction de la contrainte effective, $\sigma_{t 0}^{\prime}$ : contrainte effective verticale en tête de l'échantillon de sol, $\Delta z$ : hauteur de l'échantillon, $\gamma$ ': poids volumique déjaugé et $\gamma_{w}$ : poids volumique de l'eau.

Dans le cas du phénomène de renard, SELLMEIJER (1988) a exprimé le gradient hydraulique critique par :

$$
i_{c r}=\frac{\gamma_{p}^{\prime}}{\gamma_{w}} \operatorname{Tan} \Theta \bar{C}\left(1-0.65 \bar{C}^{0.42}\right) \text { et } \bar{C}=\frac{1}{\beta}\left(\frac{d^{2}}{K} \frac{2 d}{L}\right)^{1 / 3}
$$




\section{XIII $I^{\text {èmes }}$ Journées Nationales Génie Côtier - Génie Civil \\ Dunkerque, 2-4 juillet 2014}

avec $\gamma_{P}{ }_{P}$ : poids volumique déjaugé des particules, $\Theta$ : angle de pendage (qui relie les forces verticales et horizontales qui s’appliquent sur une particule), $K$ : perméabilité intrinsèque, $L$ : longueur de l'écoulement, $\beta$ : facteur de traînée (rapport de la surface influencée par la traînée et de la section transversale de la particule) et $d$ : diamètre d'une particule (entre $d_{65}$ et $d_{75}$, avec $d_{x}$ : ouverture du tamis au travers duquel passe $x \%$ du poids des grains du sol).

Les équations (1) et (2) sont inversement proportionnelles à la longueur de l'écoulement. Cette évolution a été confirmée par une campagne d'essais de suffusion réalisée sur des échantillons sablo argileux de trois longueurs $\Delta z$ différentes et sous macro gravité pour contrôler la contrainte effective appliquée (MAROT et al., 2012b). Donc les valeurs du gradient hydraulique critique déterminées à l'échelle d'un échantillon peuvent être plus élevées de plusieurs ordres de grandeur que la valeur à l'échelle réelle, ce qui va à l'encontre de la gestion des risques des ouvrages.

\subsection{Différentes caractérisations de la sensibilité à l'érosion d'interface}

Les interprétations des essais d'érosion d'interface les plus couramment réalisées (WAN \& FELL, 2004 ; HANSON \& COOK, 2004) sont basées sur la loi linéaire d'érosion qui s'exprime par :

$$
\dot{m}=k_{d}\left(\tau-\tau_{c}\right)
$$

avec $m$ : taux d'érosion, $k_{d}$ : coefficient d'érosion, $\tau$ : contrainte de cisaillement hydraulique, $\tau_{c}$ : contrainte seuil de cisaillement.

WAN \& FELL (2004) proposent une classification des risques de rupture de barrages en terre qui est basée sur la valeur du coefficient d'érosion mesuré par l'essai du Hole Erosion Test (HET). A partir des essais effectués à l'aide du Jet Erosion Test (JET), HANSON \& SIMON (2001) ont établi une classification qui dépend d'une part des valeurs du coefficient d'érosion et d'autre part des valeurs de la contrainte seuil de cisaillement. Afin de confronter ces deux appareillages et les classifications associées, sept sols fins naturels couvrant une large plage de variation de sensibilité à l'érosion ont été testés (REGAZZONI \& MAROT, 2013). Les résultats de cette confrontation indiquent que l'indice d'érosion ( $\left.I=-\log k_{d}\right)$ est systématiquement plus faible avec le JET ( $\left.I_{J E T}\right)$ par rapport à celui obtenu avec le HET $\left(I_{H E T}\right)$ et le rapport $I_{J E T} / I_{H E T}$ est compris entre 0,32 et 0,84 . Par ailleurs, les valeurs mesurées de contrainte seuil sont en moyenne 50 fois plus élevées avec le HET par rapport à celles mesurées avec le JET. L'étude met également en évidence des divergences de classification relative de la sensibilité des sols à l'érosion d'interface. Ainsi la classification de la sensibilité d'un sol à partir de cette approche, même par rapport à un sol de référence, risque de fortement dépendre de l'érodimètre utilisé. 


\section{Analyse énergétique pour caractériser la sensibilité des deux types d'érosion interne}

\subsection{Principe de l'analyse énergétique}

La nouvelle analyse proposée est basée sur la détermination de l'énergie dissipée par le fluide et sur la mesure de la masse érodée. Un volume $V$ de fluide, de masse $M$ possède une surface de contact $S$ avec son environnement. Cette surface est orientée par son vecteur normal, $\vec{n}$, orienté du fluide vers l'environnement. L'énergie dissipée par le fluide entre l'entrée et la sortie du système étudié peut être exprimée par (MAROT et al., 2011) :

$\frac{d E}{d t}=\frac{d}{d t} \iiint_{\text {Mass }}\left(e_{\mathrm{int}}+\frac{w^{2}}{2}+\vec{g} \vec{z}\right) d M=\frac{\partial}{\partial t} \iiint_{\text {Volume }}\left(e_{\mathrm{int}}+\frac{w^{2}}{2}+\vec{g} \vec{z}\right) \rho d V+\oiint_{S}\left(e_{\mathrm{int}}+\frac{w^{2}}{2}+\vec{g} \vec{z}\right) \cdot \rho(\vec{U} \vec{n}) \cdot d S$

et

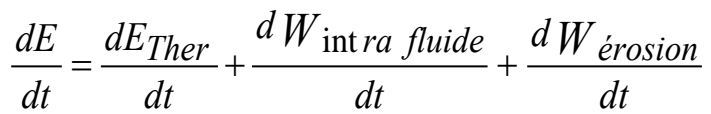

avec $E_{\text {ther }}$ : énergie thermique échangée entre le système et l'environnement, $W_{\text {intra fluide }}$ : travail dissipé par viscosité et turbulence au sein du fluide, $W_{\text {érosion }}$ : travail d'érosion à l'interface fluide-sol, $e_{i n t}$ : énergie interne du fluide, $\rho$ : masse volumique du fluide, $U$ : vitesse du fluide de composantes $(u, v, w), g$ : accélération de la pesanteur, $z$ : coordonnées.

Afin de simplifier l'équation, la température et donc l'énergie interne sont supposées constantes pour le volume considéré. Le système pouvant être considéré comme adiabatique, seul le travail mécanique entre l'entrée et la sortie du système sera considéré. L’hypothèse d'un écoulement permanent permet de négliger la variation temporelle de l'énergie cinétique et la masse volumique du fluide est supposée constante. L’équation (4) peut donc être réécrite sous la forme :

$\frac{d W_{\text {intra fluide }}}{d t}+\frac{d W_{\text {érosion }}}{d t}=\oiint_{S}\left(\frac{w^{2}}{2}+\vec{g} \vec{z}+\frac{P}{\rho}\right) \rho(\vec{U} \vec{n}) d S$

\subsection{Analyse énergétique de la suffusion}

L'équation (6) est appliquée entre la section amont $A$ et la section aval B de l'échantillon testé qui sont de même taille et parallèles (MAROT et al., 2012b). La conservation du débit et la faiblesse du nombre de Reynolds permettent d'aboutir à l'expression de la dérivée temporelle du travail d'érosion, appelée puissance d'érosion :

$$
\frac{d W_{\text {erosion }}}{d t}=\rho g \Delta z Q+Q \Delta P
$$

avec $\Delta P=P_{A}-P_{B}, \Delta z=z_{A}-z_{B}, Q:$ débit du fluide. 


\section{XIII $I^{\text {èmes }}$ Journées Nationales Génie Côtier - Génie Civil \\ Dunkerque, 2-4 juillet 2014}

\subsection{Analyse énergétique du HET}

L'équation (6) est appliquée entre les 2 points de mesures de la charge situés de part et d'autre de l'échantillon testé. La conservation du débit entre la partie à l'amont et la partie à l'aval de l'échantillon permet de supposer une vitesse moyenne constante entre ces deux points. Au cours de l'essai, l'écoulement est horizontal et dans la gamme de débit correspondant aux essais HET, la perte de charge par frottement au sein de la conduite représente $25 \%$ de la perte de charge totale mesurée lors des essais HET (MAROT et al., 2011). En conséquence, la puissance d'érosion pour un essai HET peut être exprimée par :

$\frac{d W_{\text {érosion }}}{d t}=0.25\left(P_{\text {amont }}-P_{\text {aval }}\right) Q$

avec $P_{\text {amont }}$ et $P_{\text {aval }}$ : pressions respectivement à l'amont et à l'aval de l'échantillon.

\subsection{Analyse énergétique du JET}

Dans le cas de l'essai JET, l'équation (6) est appliquée entre l'orifice du jet et le trop plein du réservoir contenant l'échantillon (MAROT et al., 2011). En comparaison avec un jet libre, un jet situé face à une interface fluide-sol subit une déviation. Nous supposons que l'érosion est principalement due à cette déviation du jet qui génère un accroissement de la contrainte de cisaillement et de grandes variations de pression. Lorsqu'un jet est face à un mur, BELTAOS \& RAJARATNAM (1974) observent que la contrainte de cisaillement au niveau du mur s'accroît linéairement en fonction de la distance à l'axe du jet $r$ jusqu'à une valeur maximale obtenue pour $r=0,14 \mathrm{~J}$, avec $J$ : profondeur par rapport à l'orifice du jet. De plus, lorsque que le rapport $r / J$ s'accroît de 0 à 0,14 , la pression au niveau du mur décroît rapidement de $90 \%$ par rapport à la valeur de la pression au centre du jet. Ainsi pour une profondeur $J$ et dans la zone définie à partir de l'axe du jet par la distance latérale $r \leq 0,14 \mathrm{~J}$, l'énergie dissipée est supposée provoquer l'érosion et la puissance correspondante s'exprime par :

$\frac{d W_{\text {erosion }}}{d t}=2 \pi \int_{0}^{0.14 J} \frac{u^{2}}{2} \rho(\vec{U} \cdot \vec{n}) r d r=\pi \rho u(0, J)^{3} \int_{0}^{0.14 J}\left[\exp \left(-0.693\left(\frac{r}{b_{u}}\right)^{2}\right)\right]^{3} r d r$

Pour $J<J_{P}, u(0, J)=u(0,0)$ avec $u(0,0)$ : vitesse initiale à l'origine du jet. Pour $J>J_{P}$, $u(0, J)$ est déterminée par le rapport $u(0,0) J_{P} / J$ proposé par HANSON \& COOK (2004).

\section{Résultats de l’analyse énergétique}

\subsection{Caractérisation de la suffusion sans effet d'échelle significatif}

Pour chaque essai de suffusion, l'énergie dissipée par le fluide par érosion peut être déterminée par intégration temporelle de la puissance d'érosion sur la durée totale de l'essai. La figure 1 représente la masse sèche d'argile érodée cumulée en fonction de l'énergie dissipée pour des essais réalisés sur des échantillons sablo-argileux de 
longueurs différentes (MAROT et al., 2012b). Une corrélation linéaire peut être exprimée entre la masse sèche d'argile érodée et l'énergie dissipée. Cette corrélation ne dépendant pas de la longueur des échantillons testés, l’analyse énergétique permet donc de s'affranchir de l'effet d'échelle induit par l'approche en gradient hydraulique.

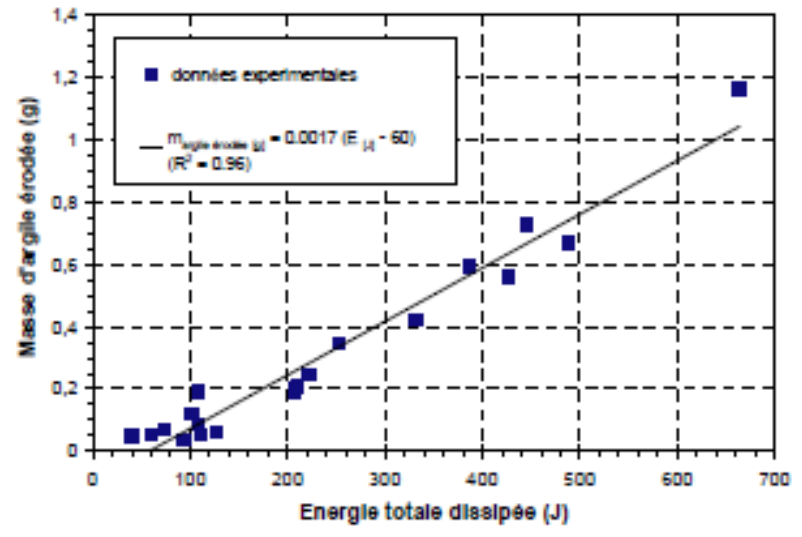

Figure 1. Masse sèche d'argile érodée versus énergie totale dissipée.

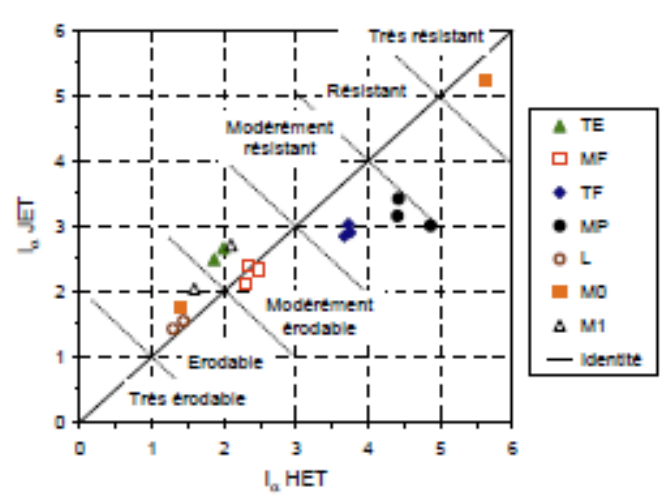

Figure 2. $I_{\alpha J E T}$ versus $I_{\alpha H E T}$ et classification de la sensibilité à l'érosion d'interface.

\subsection{Classification unique pour le HET et le JET}

L'énergie dissipée par érosion $E_{\text {érosion }}$ est déterminée pour les essais HET et JET. Un indice de résistance à l'érosion est défini par (MAROT et al., 2011) :

$$
I_{\alpha}=-\log _{10}\left(\frac{\text { masse sèche érodée }}{E_{\text {érosion }}}\right)
$$

La figure 2 représente les valeurs de l'indice $I_{\alpha}$ obtenues avec le JET ( $\left.I_{\alpha J E T}\right)$ en fonction des valeurs avec le HET $\left(I_{\alpha H E T}\right)$. A partir des valeurs de $I_{\alpha}$ pour les deux érodimètres, une corrélation linéaire peut être établie sous la forme : $I_{\alpha J E T}=0,58 I_{\alpha H E T}+0,97$ (coefficient de corrélation: $R^{2}=0,79$; REGAZZONI \& MAROT, 2013). En fonction des valeurs de l'indice de résistance à l'érosion et en prenant en compte les classifications présentes dans la littérature, six catégories de sensibilité à l'érosion d'interface sont proposées : très érodable pour $I_{\alpha}<1$, érodable pour $1 \leq I_{\alpha}<2$, modérément érodable pour $2 \leq I_{\alpha}<3$, modérément résistant pour $3 \leq I_{\alpha}<4$, résistant pour $4 \leq I_{\alpha}<5$ et très résistant pour $I_{0} \geq 5$.

Pour les deux érodimètres utilisés, une classification identique de la sensibilité à l'érosion d'interface des différents sols testés est donc obtenue grâce à l'approche énergétique. Cette analyse donne donc la possibilité de choisir l'érodimètre le plus approprié. 


\section{XIII $I^{\text {èmes }}$ Journées Nationales Génie Côtier - Génie Civil \\ Dunkerque, 2-4 juillet 2014}

\section{Détermination de la susceptibilité à la suffusion et méthodologie systématique}

\subsection{Identification des critères granulométriques adaptés}

Afin de caractériser la susceptibilité du sol à la suffusion, il convient tout d'abord d'identifier le critère granulométrique adapté à la distribution granulométrique du sol étudié. La confrontation des études comparatives de critères granulométriques menées par LI \& FANNIN (2008) et par WAN \& FELL (2008), nous permet tout d'abord de distinguer deux natures de distribution : discontinue et continue.

Dans le cas d'une distribution discontinue, seul le critère de Kézdi apparaît adapté.

Dans le cas d'une distribution continue, deux étapes successives sont effectuées. La première étape consiste à identifier la fraction fine par la valeur minimale du rapport $\mathrm{H} / \mathrm{F}$ du critère de Kenney \& Lau (avec F : pourcentage massique des grains de taille inférieure à d, $\mathrm{H}$ pourcentage massique des grains entre d et 4d). Si $\mathrm{F}$ est inférieur à 15\%, le critère de Kenney \& Lau est utilisé. Si F est supérieur à 15\% deux critères sont utilisés et leurs résultats comparés : il s’agit du critère de Kézdi et celui de Wan \& Fell.

\subsection{Principe de la méthodologie systématique}

A partir des éléments précédemment énoncés, la première étape de cette méthode consiste à choisir le critère granulométrique adapté à la distribution granulométrique du sol. Le critère choisi est alors utilisé pour vérifier si le sol étudié est potentiellement suffusif. La deuxième étape de la méthodologie a pour but d’identifier et de réaliser les essais d'érosion. Si le sol n'est pas potentiellement suffusif, il faudra s'assurer qu'il ne soit pas sensible à l'érosion d'interface. En première approche cette analyse de sensibilité peut être réalisée par mesure de quatre paramètres physiques aisément mesurables et permettant l'estimation de l'indice de résistance à l'érosion d'interface (REGAZZONI \& MAROT, 2011). Avec l'objectif d'accroître la précision de détermination de l'indice de résistance à l'érosion d'interface, un essai de Jet Erosion Test ou de Hole Erosion Test devra être réalisé. Si la susceptibilité de la suffusion est avérée, il convient de réaliser des essais pour caractériser précisément la sensibilité du sol à la suffusion. Ces essais seront réalisés pour caractériser les différentes étapes induites par la suffusion. En conséquence, ces essais seront réalisés avec un filtre aval dont l'ouverture des pores sera choisie en adéquation avec les conditions réelles de filtration. Dans le cas d'un sol provenant du noyau d'un barrage zoné, la taille des ouvertures du filtre renseignera sur la taille des plus gros grains susceptibles de migrer. Dans le cas d'un ouvrage non zoné, il conviendra de considérer la possibilité de la migration de tous les grains constituants le sol étudié. Enfin la troisième étape de la méthodologie consiste à déterminer la sensibilité du sol pour différentes étapes d'érosion avec prise en compte de l'historique de chargement hydraulique et des conditions aux limites in-situ. Ainsi l'interprétation des essais sera réalisée par analyse énergétique avec détermination de l’indice de résistance à l'érosion. 


\section{Conclusion}

Avec l'objectif de proposer une méthodologie systématique de caractérisation de la sensibilité à l'érosion interne, trois étapes complémentaires sont menées. La première consiste à choisir le critère granulométrique adapté à chaque type de distribution granulométrique pour vérifier si le sol testé est potentiellement suffusif. La deuxième étape consiste à identifier puis à réaliser des essais de sensibilité des sols aux processus de suffusion et d'érosion d'interface. La troisième étape vise à interpréter les essais avec l'analyse énergétique. Cette analyse permet de s'affranchir des effets d'échelle induits par l'approche en gradient hydraulique lors d'essais de suffusion et elle permet l'établissement d'une classification unique de sensibilité à l'érosion d'interface pour les essais HET et JET. Cette étude permet ainsi de définir une méthodologie que le praticien peut mettre en œuvre pour caractériser la sensibilité des sols aux phénomènes complexes d'érosion interne.

Les auteurs souhaitent remercier EDF CIH et la Région Pays de la Loire pour leur soutient financier dans le cadre respectivement d'un contrat de recherche et des projets régionaux R2GC et EMERMOD.

\section{Références bibliographiques}

BELTAOS S., RAJARATNAM. N. (1974). Impinging circular turbulent jets. Journal of the hydraulics division, Vol. 100(HY10), pp 1313-1328.

ERINOH (2014). Guide ERINOH, Méthodologie de caractérisation expérimentale. Coordination Chevalier C.\& Bonelli S., à paraître.

HANSON G.J., SIMON A. (2001). Erodibility of cohesive streambeds in the loess area of the midwestern U.S.A. Hydrological processes, Vol. 15, $\mathrm{N}^{\circ} 1$, pp 23-38. http://dx.doi.org/10.1002/hyp.149

HANSON G.J., COOK K.R. (2004). Apparatus, test procedures, and analytical methods to measure soil erodibility in-situ. ASAE, Vol. 20, N4, pp $455-462$.

LI M. (2008). Seepage induced instability in widely graded soils. $\mathrm{PhD}$ thesis, University of British Columbia, Vancouver, Canada. http://dx.doi.org/10.1139/T08-046

LI M., FANNIN J. (2008). Comparison of two criteria for internal stability of granular soil. Canadian Geotechnical Journal, Vol. 45, pp 1303-1309.

MAROT D., BENDAHMANE F., NGUYEN H.H. (2012a). Influence of angularity of coarse fraction grains on internal erosion process. La Houille Blanche, Int. Water $\mathrm{J}^{\mathrm{al}}$, $\mathrm{N}^{\circ}$ 6, pp 47-53. http://dx.doi.org/10.1051//hb/2012040

MAROT D., LE V. D., GARNIER J., THOREL L., AUDRAIN P. (2012b). Study of scale effect in an internal erosion mechanism. EJECE, Vol. 16(1), pp1-19. http://dx.doi.org/10.1080/19648189.2012.667203

MAROT D., REGAZZONI P.L., WAHL T. (2011). Energy based method for providing soil surface erodibility rankings. $\mathrm{J}^{\mathrm{al}}$ of Geotech. and Geoenv. Eng., Vol. 137(12), pp 1290 - 1294. 


\section{XIII $I^{\text {èmes }}$ Journées Nationales Génie Côtier - Génie Civil \\ Dunkerque, 2-4 juillet 2014}

REGAZZONI P-L, MAROT D. (2011). Investigation of interface erosion rate by Jet Erosion Test and statistical analysis. EJECE, Vol. 15(8), pp 1167-1185. http://dx.doi.org/10.3166/ejece.15.1167-1185

REGAZZONI P-L, MAROT D. (2013). A comparative analysis of interface erosion tests. Natural Hazards, Vol. 67(2), pp 937-950. http://dx.doi.org/10.1007/s11069-013-0620-3

SELLMEIJER J.B. (1988). On the mechanism of piping under impervious structures. PhD thesis, Delft University of Technology, Netherlands.

WAN C.F., FELL R. (2004). Investigation of rate of erosion of soils in embankment dams. $\mathrm{J}^{\mathrm{al}}$ of Geotech. and Geoenv. Eng., Vol. 130(4), pp 373-380. http://dx.doi.org/10.1061/(ASCE)1090-0241(2004)130:4(373)

WAN C.F., FELL R. (2008). Assessing the potential of internal instability and suffusion in embankment dams and their foundations. ${ }^{\mathrm{al}}$ of Geot. \& Geoenv. Eng., Vol. 134(3), pp 401-407. http://dx.doi.org/10.1061/(ASCE)1090-0241(2008)134:3(401) 
Thème 7 - Risques côtiers 